\title{
Supporting Information: Charge Transport in Alkali-Metal Superoxides: A Systematic First-Principles Study
}

\author{
Nicolai Rask Mathiesen, ${ }^{\#\rfloor}$ Sheng Yang, ${ }^{+\rfloor}$Juan Maria García-Lastra, ${ }^{\# *}$ Tejs Vegge, ${ }^{\#}$ and Donald J. \\ Siegel ${ }^{\mp \perp \S \Downarrow_{* *}^{*}}$ \\ $\dagger$ Department of Physics, $¥$ Mechanical Engineering Department, ${ }^{\perp}$ Materials Science \& Engineering, ${ }^{\varsigma}$ Applied Physics \\ Program, ${ }^{\sqrt{ }}$ University of Michigan Energy Institute, University of Michigan, Ann Arbor, MI 48109, USA \\ \#Department of Energy Conversion and Storage, Technical University of Denmark, Fysikvej, Building 309, 2800 Kgs \\ Lyngby, Denmark \\ *E-mail: djsiege@umich.edu. Tel.: +1 (734) 764-4808; jmgla@dtu.dk. Tel.: +45 45258213 \\ 'These authors contributed equally.
}

\section{Normalization of $+U$ in VASP}

Care should be taken in applying a $+\mathrm{U}$ correction to $p$-orbitals. In the rotationally invariant formulation, the total energy is written as ${ }^{1}$

$$
E_{\mathrm{DFT}+\mathrm{U}}=E_{\mathrm{DFT}}+\sum_{a} \frac{U}{2} \operatorname{Tr}\left(\rho^{a}-\rho^{a} \rho^{a}\right),
$$

where $E_{\mathrm{DFT}}$ is the total energy of the base functional, $\mathrm{U}$ is the Hubbard correction, and $\rho^{a}$ is the atomic orbital occupation matrix (AOOM) of atom $a$. To evaluate this expression $\rho^{a}$ is constructed from the density matrix $D_{n l m, n \prime l m}^{a}$ and the all-electron (AE) atomic orbitals $\phi_{n l}^{a}$ as $^{2}$

$$
\rho_{m m^{\prime}}^{a}=\sum_{n, n^{\prime}} D_{n l m, n^{\prime} l m^{\prime}}^{a}\left\langle\phi_{n l}^{a} \mid \phi_{n^{\prime} l}^{a}\right\rangle,
$$

where $l$ is the orbital quantum number, $m$ is the magnetic quantum numbers, and $n$ is the $n$th projector of a given orbital $l$. In principle any number of projectors can be used, however, most atomic setups include two projectors: one for a bound state and one for an unbound state. Often the integration $\left\langle\phi_{n l}^{a} \mid \phi_{n^{\prime} l}^{a}\right\rangle$ is truncated at or close to the augmentation sphere radius. This procedure for constructing the AOOM can lead to unexpected and incomparable results between different code implementations when the $+U$ correction is applied to $p$ orbitals. In contrast to more localized $d$-orbitals, for $p$-orbitals usually more than half of the wavefunction is located outside the augmentation sphere. This issue is handled by normalizing the integral of the projected $\mathrm{AE}$ atomic orbitals within the augmentation sphere, i.e. we set $\left\langle\phi_{n l}^{a} \mid \phi_{n l}^{a}\right\rangle=1$ and scale the overlaps accordingly. This normalization, which we have implemented in a customized version of VASP, is similar to the implementation in GPAW ${ }^{3}$ and sets a standard which ensures that results for a given $U$ value can be compared across codes (with small differences introduced by differentiating atomic setups). Additionally the $U$ value recovers some meaning in simple cases, such as for the splitting of the occupied and unoccupied $p$-orbitals of 
the Nitrogen atom. For a normalized $+U$ correction the splitting scales linearly with $U$ whereas for the nonnormalized correction this is not the case. ${ }^{4}$

Tilting angle and band gap

Figure $\mathrm{S} 1$ shows the energy and band gap versus $\mathrm{O}_{2}$ orientations for $\mathrm{KO}_{2}$. The optimal tilting angle is found to be at $23^{\circ}$ and $21^{\circ}$ for the $[100] /[010]$ and $[110] /[\overline{110}]$ tilting orientations respectively. In line with Kim et al. ${ }^{5}$ we see that both HSE and GGA+U predict the high symmetry structure to be a metal. However, tilting the $\mathrm{O}_{2}$ dimers breaks the orbital degeneracy and thus opens a band gap. Once the tilting angle is large enough that the band gap opens the gap continues to increase with increasing tilting angle. The band gap varies from $2.6 \mathrm{eV}$ at $\theta=5^{\circ}$ to $3.6 \mathrm{eV}$ at $\theta=35^{\circ}$. The effect of rotating the dimers is comparable with a minimal gap of $2.95 \mathrm{eV}$ when the dimers are perpendicular and a maximum gap of $3.71 \mathrm{eV}$ when they are parallel. Although the $\mathrm{O}_{2}$ dimers move in a shallow potential their orientation can indeed have a big impact on the electronic properties.
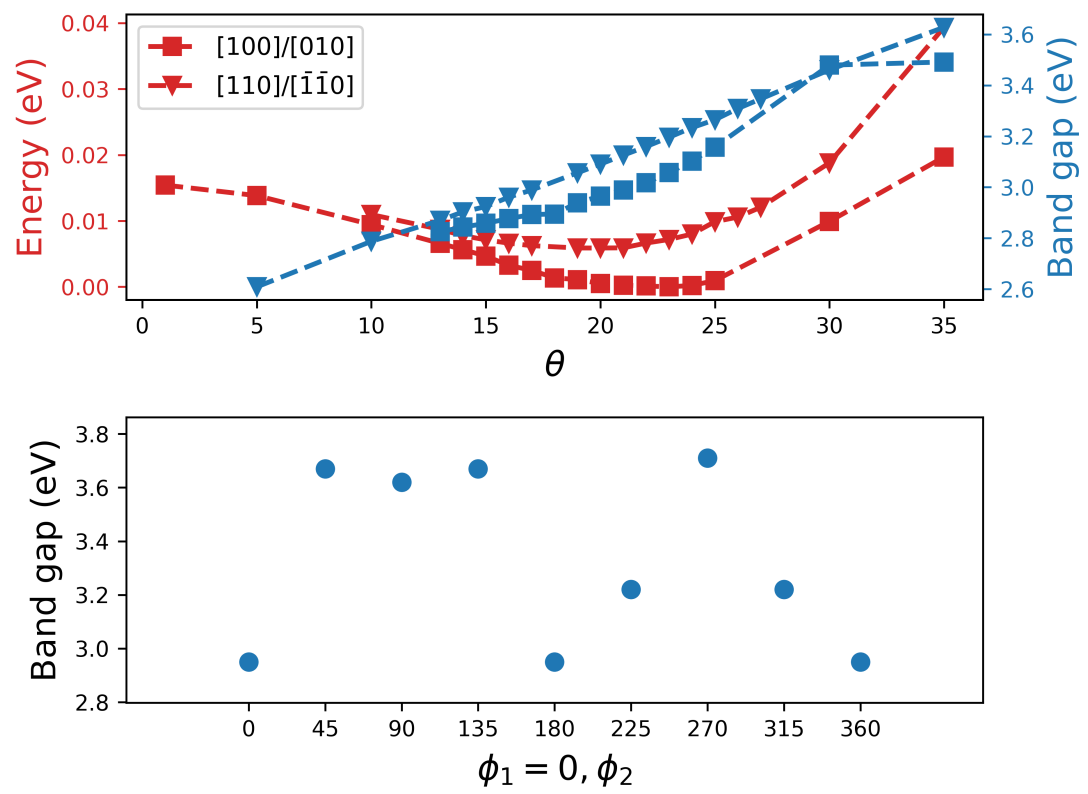

Figure S1. Top: Energy and band gap versus tilting angle for $\mathrm{KO}_{2}$. Bottom: Bandgap versus rotating one dimer in the primitive unit cell. 


\section{Comparing GGA+U and HSE functionals for $\mathrm{O}_{2}$ precession in $\mathrm{KO}_{2}$}

Figure S2 compares the energy of several dimer orientations in $\mathrm{KO}_{2}$ calculated with $\mathrm{HSE}$ and GGA+U. The two functionals agree on the overall trend. The data show that the dimer orientation predicted by GGA+U with $\phi_{1}=0$ and $\phi_{2}=90$ is also the preferred orientation with HSE. It also suggests that the barrier for rotation might be even lower according to HSE.

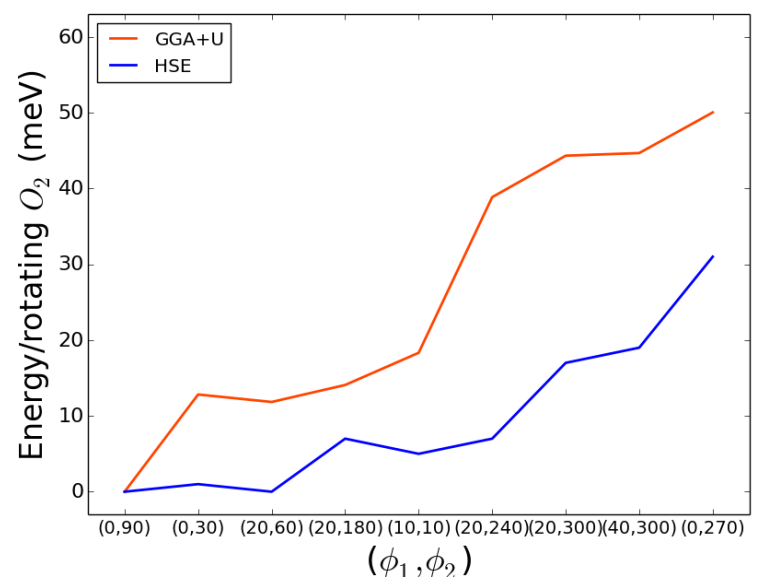

Figure S2. Comparison of HSE and GGA+U for 9 selected orientations of the $\mathrm{O}_{2}$ dimer in $\mathrm{KO}_{2}$. 


\section{Rotation of $\mathrm{O}_{2}$ dimers}

$\mathrm{LiO}_{2}$ in the marcasite structure has only two dimers in the unit cell. The tilting angle is found to minimize the energy at $\theta=50^{\circ}$, i.e. with the dimers oriented as in the $\mathrm{NaO}_{2}$ marcasite phase. Figure S3 defines $\theta, \phi_{1}$, and $\phi_{2}$ for $\mathrm{LiO}_{2}$. Figure $\mathrm{S} 4$ shows the potential energy map for $\mathrm{O}_{2}$ orientations in $\mathrm{LiO}_{2}$. The optimal structure is found to be at $\theta=50, \phi_{1}=0$, and $\phi_{2}=0$.

a)

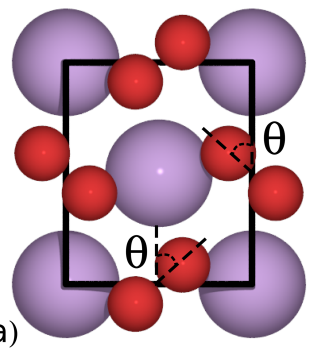

b)

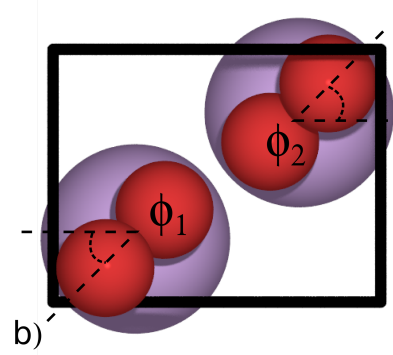

Figure S3. $\mathrm{LiO}_{2}$ viewed from a) the [001] direction and b) [010].

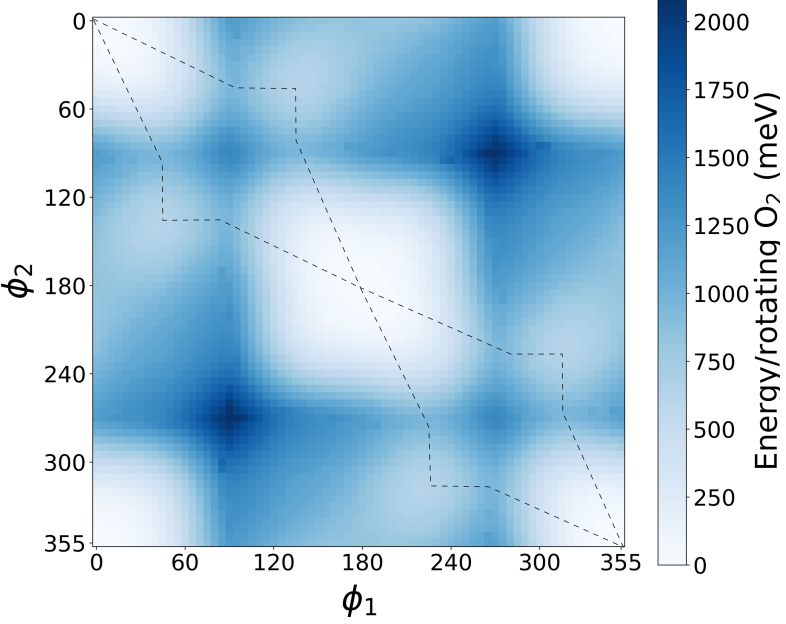

Figure S4. Potential energy map of $\mathrm{O}_{2}$ orientations in $\mathrm{LiO}_{2}$ with a fixed $\theta=50^{\circ}$. The black dashed lines mark the minimum energy path for a full precession the dimers. Only the points where $\phi_{1} \in$ $[0,90]$ and $\phi_{2} \in[0,355]$ have been calculated; the remainder of the map is filled by symmetry considerations.

$\mathrm{NaO}_{2}$ is in the pyrite structure with four dimers in the unit cell where the dimers point in the [111], [111], [1111], and [1111] directions. To limit the search space we modify the structure to be marcasite-like by rotation two of the dimers so that they align in pairs and oppose additional constraints. That is, we rotate the dimer

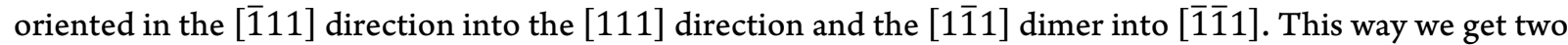
layers with parallel dimers that can be rotated together as shown in Figure s4. The tilting angle is found to minimize the energy at $\theta=55^{\circ}$, i.e. with the dimers oriented in the [110] and [110] directions. Figure s5 shows the potential energy map for $\mathrm{O}_{2}$ orientations in $\mathrm{NaO}_{2}$. The optimal structure is found to be at $\theta=55$, $\phi_{1}=10$, and $\phi_{2}=10$. We use a $4 \times 4 \times 4$ k-point grid for calculations involving $\mathrm{NaO}_{2}$. 


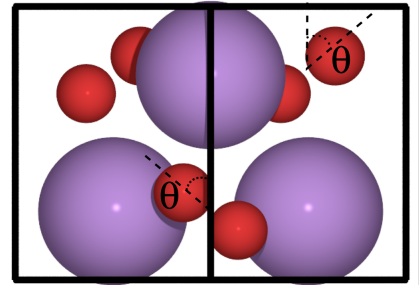

a)

Figure S5. $\mathrm{NaO}_{2}$ viewed from a) the [110] direction and $\mathrm{b}$ ) [001].

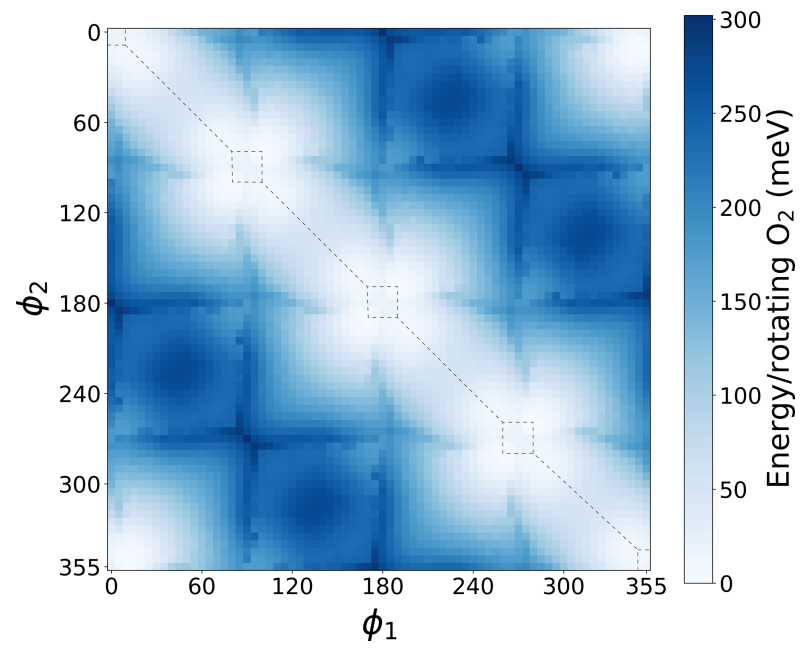

Figure S6. Potential energy map of $\mathrm{O}_{2}$ orientations in $\mathrm{NaO}_{2}$ with a fixed $\theta=55^{\circ}$. The black dashed lines mark the minimum energy path for a full precession the dimers. Only the points where $\phi_{1} \in$ $[0,45]$ and $\phi_{2} \in[0,355]$ have been calculated; the remainder of the map is filled by symmetry considerations. 


\section{Formation energies}

Figures S7 and S8 show the calculated defect formation energies in $\mathrm{LiO}_{2}$ and $\mathrm{KO}_{2}$, respectively. The slope of each line indicates the charge state for the respective defect species: a positive (negative) line indicates a positively (negatively) charged defect. The zero of the $\mathrm{x}$-axis corresponds to the valence band maximum (VBM), while the maximum value represents the conduction band minimum (CBM). The vertical dotted line gives the position of the Fermi energy, which is established by the charge neutrality condition.

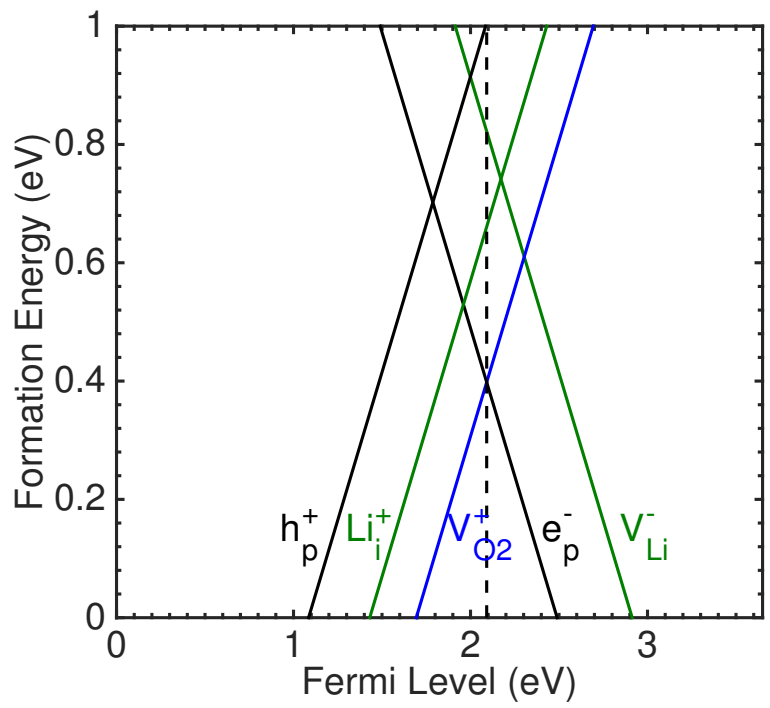

Figure S7. Defect formation energy of the $\mathrm{O}$ vacancy, Li vacancy and interstitials, electron polarons and hole polarons obtained using the HSE $\alpha(\alpha=0.48)$ hybrid functional in $\mathrm{LiO}_{2}$.

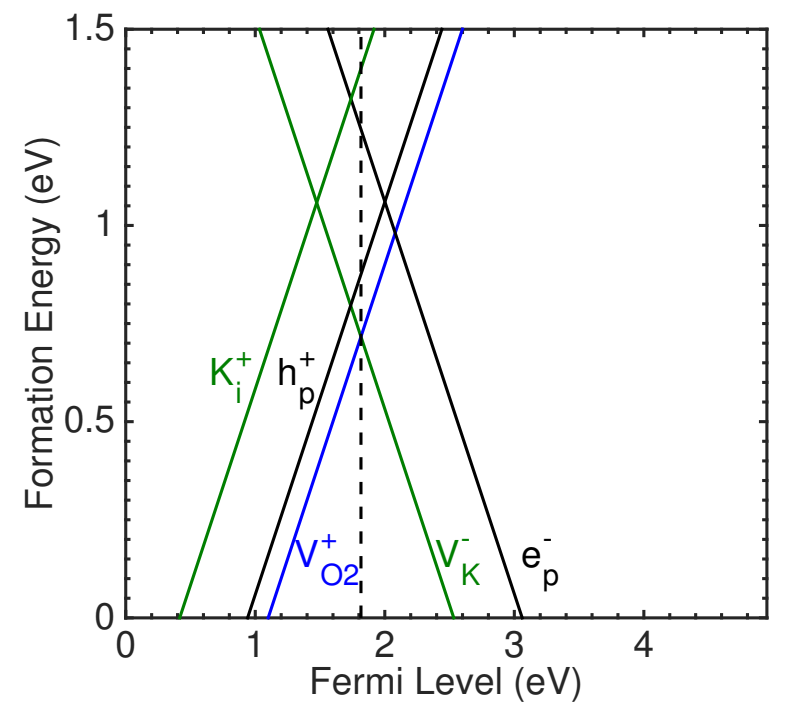

Figure S8. Defect formation energy of the $\mathrm{O}$ vacancy, $\mathrm{K}$ vacancy and interstitials, electron polarons and hole polarons obtained using the $\mathrm{HSE} \alpha(\alpha=0.48)$ hybrid functional in $\mathrm{KO}_{2}$. 


\section{Calculated formation energies and equilibrium concentrations of charged defects}

Table S1. Defect formation energies $\left(E_{f}\right)$ and concentrations $(C)$ in $\mathrm{LiO}_{2}$.

\begin{tabular}{c|c|c}
\hline Defects & $\mathrm{E}_{\mathrm{f}}(\mathrm{eV})$ & $\mathrm{C}\left(\mathrm{cm}^{-3}\right)$ \\
\hline \hline $\mathrm{e}_{\mathrm{p}}{ }^{-}$ & 0.40 & $7 \times 10^{15}$ \\
$\mathrm{~h}_{\mathrm{p}}{ }^{+}$ & 1.00 & $5 \times 10^{5}$ \\
$\mathrm{~V}_{\mathrm{Li}}{ }^{-}$ & 0.82 & $6 \times 10^{8}$ \\
$\mathrm{~V}_{\mathrm{O}^{+}}{ }^{+}$ & 0.40 & $7 \times 10^{15}$ \\
$\mathrm{Li}_{\mathrm{i}}{ }^{+}$ & 0.66 & $6 \times 10^{11}$
\end{tabular}

Table S2. Defect formation energies $\left(E_{f}\right)$ and concentrations $(C)$ in $\mathrm{KO}_{2}$

\begin{tabular}{c|c|c}
\hline Defects & $\mathrm{E}_{\mathrm{f}}(\mathrm{eV})$ & $\mathrm{C}\left(\mathrm{cm}^{-3}\right)$ \\
\hline $\mathrm{e}_{\mathrm{p}}{ }^{-}$ & 1.24 & $2 \times 10^{1}$ \\
$\mathrm{~h}_{\mathrm{p}}{ }^{+}$ & 0.88 & $4 \times 10^{7}$ \\
$\mathrm{~V}_{\mathrm{K}}{ }^{-}$ & 0.72 & $2 \times 10^{10}$ \\
$\mathrm{~V}_{\mathrm{O}^{+}}{ }^{+}$ & 0.72 & $2 \times 10^{10}$ \\
$\mathrm{~K}_{\mathrm{i}}^{+}$ & 1.40 & $6 \times 10^{-2}$
\end{tabular}




\section{Migration barriers}

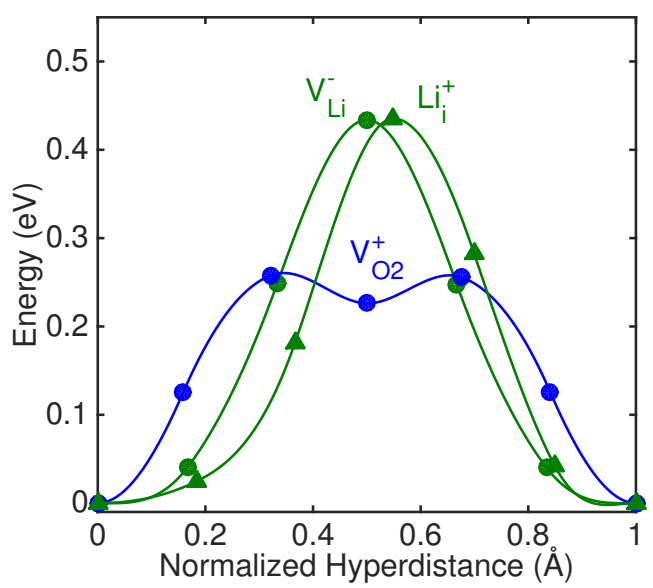

(a)

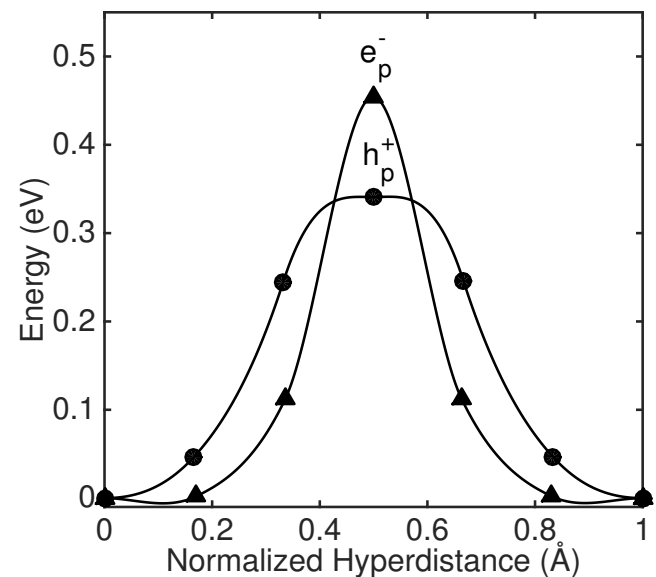

(b)

Figure S9. Energy barriers calculated using the NEB method for (a) ionic charge carriers and (b) polarons in $\mathrm{LiO}_{2}$. Blue curves refer to positive lithium interstitials $\left(\mathrm{Li}_{\mathrm{i}}^{+}\right)$, green curves represent negative potassium vacancies $\left(\mathrm{V}_{\mathrm{Li}^{-}}{ }^{-}\right)$, and black curves are polarons $\left(\mathrm{e}_{\mathrm{p}}{ }^{-}, \mathrm{h}_{\mathrm{p}}{ }^{+}\right)$.

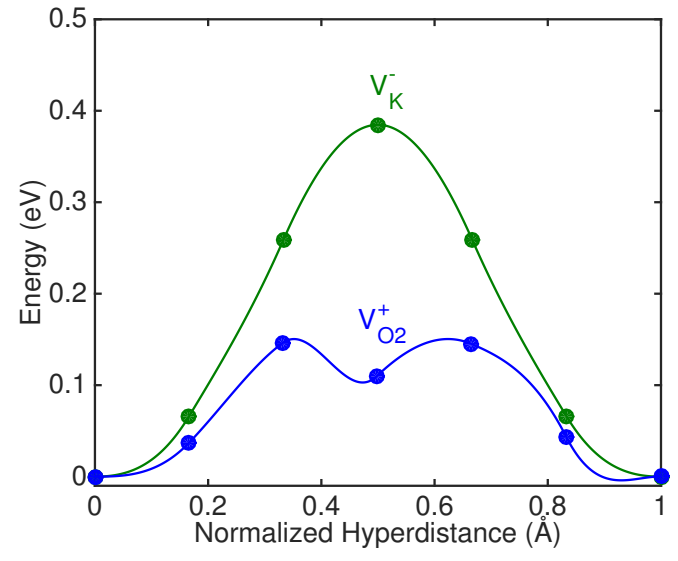

(a)

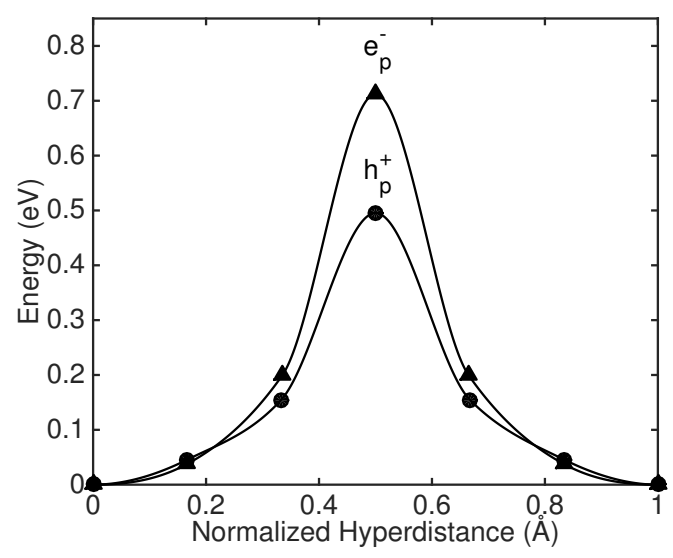

(b)

Figure S10. Energy barriers calculated using the NEB method for (a) ionic charge carriers and (b) polarons in $\mathrm{KO}_{2}$. Blue curves refer to positive potassium interstitials $(\mathrm{Ki}+)$, green curves represent negative potassium vacancies (VK-), and black curves are polarons (ep-, hp+). 


\section{References}

(1) Dudarev, S. L.; Botton, G. A.; Savrasov, S. Y.; Humphreys, C. J.; Sutton, A. P. Electron-EnergyLoss Spectra and the Structural Stability of Nickel Oxide: An LSDA+U Study. Phys. Rev. B 1998, 57 (3), 1505-1509.

(2) Rohrbach, A.; Hafner, J.; Kresse, G. Molecular Adsorption on the Surface of Strongly Correlated Transition-Metal Oxides: A Case Study for CO/NiO(100). Phys. Rev. B 2004, 69 (7), 075413.

(3) Enkovaara, J.; Rostgaard, C.; Mortensen, J. J.; Chen, J.; Dułak, M.; Ferrighi, L.; Gavnholt, J.; Glinsvad, C.; Haikola, V.; Hansen, H. a; et al. Electronic Structure Calculations with GPAW: A Real-Space Implementation of the Projector Augmented-Wave Method. J. Phys. Condens. Matter 2010, $22(25), 253202$.

(4) GPAW DFT+U https://wiki.fysik.dtu.dk/gpaw/tutorials/hubbardu/hubbardu.html.

(5) Kim, M.; Min, B. I. Temperature-Dependent Orbital Physics in a Spin-Orbital-Lattice-Coupled $2 \mathrm{p}$ Electron Mott System: The Case of K O 2. Phys. Rev. B - Condens. Matter Mater. Phys. 2014, 89 (12), 1211065. 